\title{
Note: An exact scaling relation for truncatable free energies of polydisperse hard-sphere mixtures
}

\author{
Andrés Santo:* \\ Departamento de Física, Universidad de Extremadura, Badajoz, E-06071, Spain
}

(Dated: October 29, 2018)

\begin{abstract}
A theoretical model for polydisperse systems of hard spheres is said to be truncatable when the excess free energy depends on the size distribution through a finite number $K$ of moments. This Note proves an exact scaling relation for truncatable free energies, which allows to reduce the effective degrees of freedom to $K-2$ independent combinations of the moments.
\end{abstract}

Let us consider a polydisperse hard-sphere fluid mixture in $d$ dimensions with number density $\rho=N / V$, where $N$ is the total number of particles contained in a volume $V$. The number of particles with a diameter between $\sigma$ and $\sigma+\mathrm{d} \sigma$ is $N x(\sigma) \mathrm{d} \sigma$, so that $\int_{0}^{\infty} \mathrm{d} \sigma x(\sigma)=1$. The moments of the size distribution are

$$
M_{n} \equiv \int_{0}^{\infty} \mathrm{d} \sigma x(\sigma) \sigma^{n} .
$$

The particular case of a discrete $s$-component mixture is included as $x(\sigma)=\sum_{i=1}^{s} x_{i} \delta\left(\sigma-\sigma_{i}\right)$ with $\sum_{i=1}^{s} x_{i}=1$.

Let $a^{\operatorname{ex}}[\rho, x(\sigma)]$ be the excess free energy per particle of the system in units of $k_{B} T$, where $k_{B}$ is the Boltzmann constant and $T$ is the absolute temperature. It is given by

$$
a^{\operatorname{ex}}[\rho, x(\sigma)]=-N^{-1} \ln Q_{N}(V),
$$

where

$$
Q_{N}(V)=V^{-N} \int \mathrm{d} \mathbf{r}_{1} \ldots \int \mathrm{d} \mathbf{r}_{N} \prod_{1 \leq i<j \leq N} \Theta\left(r_{i j}-\frac{\sigma_{i}+\sigma_{j}}{2}\right)
$$

is the configuration integral, $\Theta(x)$ being the Heaviside step function.

We now suppose that the mixture consists of a finite number $N_{0}=x_{0} N$ of point particles (i.e., $\sigma_{i}=0$ for $\left.1 \leq i \leq N_{0}\right)$ plus $N^{\prime}=N-N_{0}$ particles $\left(N_{0}+1 \leq j \leq N\right)$ with a certain (continuous or discrete) size distribution $x^{\prime}(\sigma)$. Thus,

$$
x(\sigma)=x_{0} \delta(\sigma)+\left(1-x_{0}\right) x^{\prime}(\sigma) .
$$

The number density of the "bare" mixture is $\rho^{\prime}=$ $N^{\prime} / V=\left(1-x_{0}\right) \rho$ and its size moments are related to those of the original composite mixture by

$$
M_{n}^{\prime}=\int_{0}^{\infty} \mathrm{d} \sigma x^{\prime}(\sigma) \sigma^{n}=\frac{M_{n}}{1-x_{0}}, \quad n \geq 1 .
$$

Setting $\sigma_{i}=0\left(1 \leq i \leq N_{0}\right)$ in Eq. (3) we get

$$
Q_{N}(V)=\left(1-V^{\prime} / V\right)^{N_{0}} Q_{N^{\prime}}(V),
$$

where $V^{\prime}=v_{d} N^{\prime} M_{d}^{\prime}=v_{d} N M_{d}$ is the volume excluded by the $N^{\prime}$ normal particles, $v_{d}=(\pi / 4)^{d / 2} / \Gamma(1+d / 2)$ being the volume of a $d$-dimensional sphere of unit diameter, and $Q_{N^{\prime}}(V)$ is the configuration integral of the bare mixture. In the derivation of Eq. (6) one first integrates over the positions of the point particles, obtaining $\left(V-V^{\prime}\right)^{N^{\prime}}$; the remaining integration over the normal particles is then the same as in the system without point particles. Therefore, in the special case (4), Eq. (2) becomes

$a^{\mathrm{ex}}[\rho, x(\sigma)]=-x_{0} \ln \left(1-v_{d} \rho M_{d}\right)+\left(1-x_{0}\right) a^{\mathrm{ex}}\left[\rho^{\prime}, x^{\prime}(\sigma)\right]$.

Let us now go back to the general polydisperse case and assume a model free energy with "truncatable" structure, $\stackrel{1-3}{-3}$ i.e., the excess free energy $a^{\operatorname{ex}}[\rho, x(\sigma)]$ depends on the size distribution $x(\sigma)$ only through a finite number $K$ of moments $\left\{M_{1}, M_{2}, \ldots, M_{K}\right\}$. Dimensional analysis requires that the dependence of $a^{\mathrm{ex}}$ on $\rho$ and $\left\{M_{1}, M_{2}, \ldots, M_{K}\right\}$ takes place through the dimensionless combinations $\eta$ and $\left\{m_{2}, \ldots, m_{K}\right\}$, where $\eta \equiv v_{d} \rho M_{d}$ is the packing fraction and $m_{n} \equiv M_{n} / M_{1}^{n}$ are rescaled moments. Therefore, the truncatability hypothesis can be written as

$$
a^{\mathrm{ex}}[\rho, x(\sigma)]=a^{\mathrm{ex}}\left(\eta ; m_{2}, \ldots, m_{K}\right) .
$$

It is important to bear in mind that Eq. (8) is not a rigorous property. For instance, Blaak ${ }^{\underline{4}}$ has exactly evaluated the fourth virial coefficient $B_{4}\left(\sigma_{i}, \sigma_{j}, \sigma_{k}, \sigma_{\ell}\right)$ for $d=3$ when the four diameters are such that the smallest sphere (say $\ell$ ) fits in the inner space made by the other three spheres being tangent to each other. The analytic expression of $B_{4}\left(\sigma_{i}, \sigma_{j}, \sigma_{k}, \sigma_{\ell}\right)$ is given as a linear combination of terms of the form $\sigma_{i}^{q_{1}} \sigma_{j}^{q_{2}} \sigma_{k}^{q_{3}} \sigma_{\ell}^{q_{4}}$ with $q_{1}+q_{2}+q_{3}+q_{4}=9$. However, while $q_{1}, q_{2}$, and $q_{3}$ are always not larger than 3 , terms with up to $q_{4}=9$ are present. This shows that the exact $B_{4}$ is, in contrast to what claimed in other works, $\frac{5}{5}$ incompatible with Eq. (8), unless $K \geq 9$. Notwithstanding this, the truncatability hypothesis (8) is crucial from a practical point of view to reduce from functional to algebraic the phase transition problem in polydisperse systems. $\stackrel{2}{2}$ Moreover, this hypothesis (with $K=3$ ) has recently received numerical support from simulation data of three-dimensional polydisperse mixtures, $\frac{6}{-}$ even for metastable states.

The objective now is to prove that, once the ansatz (8) is assumed, the exact relationship (7) imposes a constraint under the form of a scaling law for $a^{\mathrm{ex}}\left(\eta ; m_{2}, \ldots, m_{K}\right)$. First, note that, in the case of Eq. 
(4), Eq. (5) gives

$$
m_{n}^{\prime}=\left(1-x_{0}\right)^{n-1} m_{n}, \quad n \geq 2 .
$$

Next, assuming Eq. (8) and making use of Eq. (7) one obtains

$$
a^{\mathrm{ex}}\left(\eta ; m_{2}, m_{3}, \ldots, m_{K}\right)+\ln (1-\eta)=\lambda\left[a^{\mathrm{ex}}\left(\eta ; \lambda m_{2}, \lambda^{2} m_{3}, \ldots, \lambda^{K-1} m_{K}\right)+\ln (1-\eta)\right],
$$

where $\lambda=1-x_{0}$. The scaling property (10) is the main result of this Note. It implies the equivalent form

$$
a^{\mathrm{ex}}\left(\eta ; m_{2}, m_{3}, \ldots, m_{K}\right)=-\ln (1-\eta)+\frac{1}{m_{2}} \mathcal{A}\left(\eta ; \frac{m_{3}}{m_{2}^{2}}, \ldots, \frac{m_{K}}{m_{2}^{K-1}}\right),
$$

where the scaling function $\mathcal{A}$ remains undetermined. In the special case of a one-component system, $m_{n}=1$ and thus

$$
\mathcal{A}(\eta ; 1, \ldots, 1)=a_{\text {pure }}^{\text {ex }}(\eta)+\ln (1-\eta),
$$

where $a_{\mathrm{pure}}^{\mathrm{ex}}(\eta)$ is the excess free energy of the pure fluid. Scaling relations similar to (10) and (11) can be obtained for the compressibility factor $Z \equiv p / \rho k_{B} T$, where $p$ is the pressure, by the thermodynamic relation $Z=1+$ $\eta \partial a^{\mathrm{ex}} / \partial \eta$.

Equation (11) constrains possible theoretical models with truncatable structure. In fact, it represents a significant reduction in the number of "degrees of freedom" of the excess free energy of a polydisperse HS fluid. The exact free energy $a^{\text {ex }}$ is a functional of the size distribution $x(\sigma)$ and thus it has an infinite number of degrees of freedom. The truncatability hypothesis reduces the number of independent variables to the first $K$ moments $\left\{M_{n}, n=1, \ldots, K\right\}$, apart from the number density $\rho$. Dimensional analysis trivially reduces that number to $K-1$ dimensionless moments $\left\{m_{n}, n=2, \ldots, K\right\}$, as indicated in Eq. (8). Further, Eq. (11) makes explicit the dependence on $m_{2}$, thus reducing the effective number of independent quantities to $K-2$ ratios $m_{n} / m_{2}^{n-1}$ $(n=3, \ldots, K)$.

The exact excess free energy in the one-dimensional case $(d=1)$ is $a^{\mathrm{ex}}=-\ln (1-\eta)$ and thus it trivially verifies Eq. (111) with $\mathcal{A}=0$. The exact result for hard disks $(d=2)$ is not known. However, assuming truncatability with $K=2$, Eqs. (11) and (12) imply

$$
a^{\mathrm{ex}}\left(\eta ; m_{2}\right)=-\ln (1-\eta)+\frac{1}{m_{2}}\left[a_{\text {pure }}^{\mathrm{ex}}(\eta)+\ln (1-\eta)\right] .
$$

This result agrees with that derived by independent methods ${ }^{7-\underline{9}}$ and includes Jenkins and Mancini's equation of state $\frac{10}{}$ as a particular case. Finally, let us consider the three-dimensional case $(d=3)$ with $K=3$. Now the scaling function $\mathcal{A}(\eta ; y)$ depends on the ratio $y=m_{3} / m_{2}^{2}$ but otherwise it is arbitrary. Let us assume the explicit functional form $\mathcal{A}(\eta ; y)=y^{-2}\left[\mathcal{A}_{0}(\eta)+\mathcal{A}_{1}(\eta) y\right]$. Equation (12) then implies that $\mathcal{A}_{0}(\eta)=a_{\text {pure }}^{\text {ex }}(\eta)+\ln (1-\eta)-$ $\mathcal{A}_{1}(\eta)$. Therefore, Eq. (11) yields

$$
\begin{aligned}
a^{\mathrm{ex}}\left(\eta ; m_{2}, m_{3}\right)= & -\ln (1-\eta)+\frac{m_{2}^{3}}{m_{3}^{2}}\left[a_{\mathrm{pure}}^{\mathrm{ex}}(\eta)+\ln (1-\eta)\right] \\
& +\left(\frac{m_{2}}{m_{3}}-\frac{m_{2}^{3}}{m_{3}^{2}}\right) \mathcal{A}_{1}(\eta),
\end{aligned}
$$

where the function $\mathcal{A}_{1}(\eta)$ is not constrained by Eq. (12). Equation (14) with $\mathcal{A}_{1}(\eta)=3 \eta /(1-\eta)$ has been derived by a different route $9.11,12$ and includes, as particular cases, the Scaled Particle Theory $\underline{13}-18$ the virial Percus-Yevick,,$\frac{19}{\underline{2}}$ and the Boublík-Mansoori-CarnahanStarling-Leland ${ }^{20.21}$ equations of state. More generally, Eq. (14) with free $\mathcal{A}_{1}(\eta)$ or Eq. (11) with $K=3$ and free $\mathcal{A}(\eta, y)$ can be useful to infer the equation of state of the pure system in the metastable region from measurements made on multi-component systems. $\underline{22}$

The author is grateful to two anonymous reviewers for their constructive comments. Financial support from the Spanish Government through Grant No. FIS2010-16587 and from the Junta de Extremadura (Spain) through Grant No. GR10158 (partially financed by FEDER funds) is acknowledged.
* Electronic address: andres@unex.es URL: http://www.unex.es/eweb/fisteor/andres/

1 J. A. Gualtieri, J. M. Kincaid, and G. Morrison, J. Chem. Phys. 77, 521 (1982).
2 P. Sollich, P. B. Warren, and M. E. Cates, Adv. Chem. Phys. 116, 265 (2001), cond-mat/0003084.

3 P. Sollich, J. Phys.: Cond. Matt. 14, R79 (2002).

${ }^{4}$ R. Blaak, Mol. Phys. 95, 695 (1998). 
${ }^{5}$ P. Bartlett, Mol. Phys. 97, 685 (1999).

6 V. Ogarko and S. Luding, J. Chem. Phys. 136, 124508 (2012).

7 A. Santos, S. B. Yuste, and M. López de Haro, Mol. Phys. 96, 1 (1999).

8 A. Santos, S. B. Yuste, and M. López de Haro, J. Chem. Phys. 117, 5785 (2002).

9 M. López de Haro, S. B. Yuste, and A. Santos, in Theory and Simulation of Hard-Sphere Fluids and Related Systems, edited by A. Mulero (Springer, Berlin, 2008), vol. 753 of Lectures Notes in Physics, pp. 183-245.

10 J. T. Jenkins and F. Mancini, J. Appl. Mech. 54, 27 (1987).

11 A. Santos, S. B. Yuste, and M. López de Haro, J. Chem. Phys. 123, 234512 (2005).

12 M. López de Haro, S. B. Yuste, and A. Santos, Mol. Phys. 104, 3461 (2006).

13 H. Reiss, H. L. Frisch, and J. L. Lebowitz, J. Chem. Phys.
31, 369 (1959).

14 E. Helfand, H. L. Frisch, and J. L. Lebowitz, J. Chem. Phys. 34, 1037 (1961).

15 J. L. Lebowitz, E. Helfand, and E. Praestgaard, J. Chem. Phys. 43, 774 (1965).

16 M. Mandell and H. Reiss, J. Stat. Phys. 13, 113 (1975).

17 Y. Rosenfeld, J. Chem. Phys. 89, 4272 (1988).

18 M. Heying and D. Corti, J. Phys. Chem. B 108, 19756 (2004).

19 J. L. Lebowitz, Phys. Rev. 133, A895 (1964).

20 T. Boublík, J. Chem. Phys. 53, 471 (1970).

21 G. A. Mansoori, N. F. Carnahan, K. E. Starling, and J. T. W. Leland, J. Chem. Phys. 54, 1523 (1971).

22 A. Santos, S. B. Yuste, and M. López de Haro, J. Chem. Phys. 135, 181102 (2011). 